\title{
QUADRICEPS STRENGTH AND FUNCTIONAL PERFORMANCE FOLLOWING ANTERIOR CRUCIATE LIGAMENT RECONSTRUCTION IN PROFESSIONAL FOOTBALLERS, AT TIME OF RETURN TO SPORT
}

\begin{abstract}
Poor quadriceps strength has been associated with poor outcome following anterior cruciate ligament reconstruction (ACLR); this study aims to assess quadriceps strength, muscle inhibition and hop test performance in professional soccer players after ACLR. Fifteen professional soccer players (age $22.3 \pm 3.1$ years, body mass $81.0 \pm 11.5 \mathrm{~kg}$, height $1.75 \pm 0.1 \mathrm{~m}$ ) who had undergone ACLR participated. Isometric, eccentric and concentric quadriceps strength was assessed, along with quadriceps inhibition and single and cross-over hop performance, at the time of return to full time unrestricted play. In comparison to the uninjured leg, the ACLR leg demonstrated large significant $(d \geq 0.84, p<0.01)$ deficits in isometric, eccentric and concentric quadriceps strength, quadriceps inhibition and hop distance. Over $80 \%$ of the players failed to exceed the limb symmetry criteria of $\geq 90 \%$ for strength tests, although $75 \%$ of the cohort passed the $\geq 90 \%$ criteria for hop tests. The outcome from ACLR in professional footballers who received full time intensive rehabilitation has not previously been reported in detail. There were significant deficits in quadriceps strength at the time of return to sport, whilst hop testing, a commonly used outcome measure, failed to show the same levels of deficit. These deficits in quadriceps function may have implications for the development of ongoing knee symptoms and risk of future ACL injury. If this proves to be the case then it would appear that greater attention should be paid to re-establishing full quadriceps strength prior to returning to unrestricted sporting activity.
\end{abstract}

Key terms: inhibition; asymmetry; concentric; eccentric; isometric; 


\section{QUADRICEPS STRENGTH AND FUNCTIONAL PERFORMANCE FOLLOWING ACLR IN} PROFESSIONAL FOOTBALLERS AT TIME OF RETURN TO SPORT

\section{Introduction}

A recent systematic review of outcome from anterior cruciate ligament reconstruction (ACLR) in elite sports people reported pooled return to sport (RTS) rate was 83\% (95\% confidence interval 77-88\%) (14). Six out of nine studies included in the review, which had a non-injured control group found no deterioration in athletic performance following ACLR in elite sports performers. When assessing return to elite soccer Walden et al. (21) reported greater than $90 \%$ of professional football players returned to play, with $2 / 3$ still playing at the same level 3 years post-surgery. Zaffagnini et al. (25) reported a similar level of return (95\%) with $71 \%$ still playing at the same level, whilst Erickson et al. (8) reported a slightly lower level of return (77\%). Unsurprisingly, these levels of return to sport are higher than those reported for non-elite/professional populations with Ardern et al. (2) reporting the figure to be around 55\%. The reasons for the disparity appear to be obvious, full time professionally supervised rehabilitation and higher baseline (pre-operative) levels of fitness and strength, alongside the financial imperative to return to play. These reports on return to play come from retrospective audits of injury data, what is current lacking in the literature, is data on the physical status of these athletes when they return to play, and if they have superior physical qualities compared to those previously reported for non-elite patients which have supported these achievements.

Individuals who have undergone ACLR frequently been found to have deficits in quadriceps activation (level of inhibition) and rate of force development (RFD) which can persist for greater than 1 to 2 years post-surgery $(3,9)$. These deficits in muscle function (activation, strength and RFD) have been linked to decreased performance both in the sporting environment and also in activities of daily living and quality of life $(7,9)$. Furthermore, poor muscle function has been linked to poor movement quality during landing tasks, walking, running and cutting performance which exposes the athlete to increased risk of further ACL injury (12) and osteoarthritis $(1,7)$. What is currently unclear in the literature is if the higher levels of RTS in professional footballers could be attributed to overcoming these deficits. The first stage in possibly improving the outcome for non-elite ACLR patients then might be to understand the performance characteristics and physical qualities of elite athletes at RTS. Then from this information inform and define goals for rehabilitation for all ACLR patients. 
Logerstedt et al. (16) commented that both short-term and long-term outcomes after ACLR require attention and action, because of the relatively poor outcomes with less than $55 \%$ of non-elite athletes making a full recovery (2) and greater than 50\% developing significant osteoarthritis (OA) within a decade of surgery (6). Despite the relative advances in surgical techniques in the last 10 years the outcomes for the patients have remained unchanged (20). The rehabilitation undertaken by the patients post reconstructive surgery is regarded as equally important as the surgery itself in defining the patient's outcome (18), but this has altered relatively little in this period and no consensus still exists on the optimal strategies to rehabilitate these patients post ACLR (11).

Culvenor et al. (7) clearly identified, in their review, the relationship between poor quadriceps strength and the increased risk of symptomatic and functional deterioration of the knee during activities of daily living and sportrecreational activities. Furthermore, persistent quadriceps dysfunction, both in terms of strength and activation pattern, following ACLR has been identified as a risk factor for re-injury, contralateral knee joint injury and premature progression to degenerative knee joint changes $(1,6,7)$. The absolute levels of quadriceps strength (strength normalized to bodyweight) as opposed to limb symmetry index are rarely reported in the literature on outcome from ACLR. The most frequently reported variable is the limb symmetry index (LSI), thus making it difficult to understand the absolute levels of quadriceps performance. One of the few papers reporting strength found it to be a more significant predictor of outcome than LSI (19). The use of the contralateral leg as a performance comparison using metrics such as limb symmetry index (LSI) has been questioned, because it may underestimate the true level of deficit, because the contralateral leg of an ACLR patient is often significantly weaker when compared to a control limb in non-injured individuals. (5).

The aim of this study, therefore, was to assess the concentric, eccentric and isometric quadriceps strength, and level of inhibition (central activation ratio (CAR)), of elite professional footballers at the time of RTS, to identify their level of performance in relation to their quadriceps muscles. Alongside direct measures of quadriceps performance standard hop tests were assessed as a proxy for functional performance $(12,13,18)$ As these athletes have returned to sport it may then provide an insight into the physical qualities (measureable performance metrics) required to return to sport. It was hypothesized that these athletes would show LSI $>90 \%$ and not have significant performance deficits between limbs, due to lower levels of strength in the ACLR limb.

\section{Method}


An observational cross-sectional design was used to determine the level of quadriceps strength and central activation ratio, of professional soccer players, at the time of return to sport, and to compare between affected and unaffected limbs to determine if any asymmetry was evident.

91

\section{Participants}

Fifteen full-time professional footballers (age $22.3 \pm 3.1$ years, body mass $81.0 \pm 11.5 \mathrm{~kg}$, height $1.75 \pm 0.1 \mathrm{~m}$, and a global KOOS questionnaire score of $89.9 \pm 5.1$ at time of assessment), playing for clubs in the English premiership or championship divisions, who had undergone an ACLR participated in the study. Participants were recruited via orthopaedic surgeons or directly from their football teams, following an invitation letter to participate in the study. An initial screening of the volunteers was undertaken to exclude any individuals who had received more than primary ACL reconstructive surgery. Assessment was performed on all volunteers who met the inclusion criteria, between the period January 2015 - February 2017 (24 months). All participants had undergone ACL reconstruction (time since surgery $7.8 \pm 1.3$ months). All participants had been medically cleared to return to sport and undertaken and passed functional return to play testing at their clubs and all their rehabilitation had been undertaken on a full-time basis within their professional club environment supervised by a sports physiotherapist, sports physician and Orthopaedic surgeon. Ten of the 15 had received a hamstring autograft and 5 had received a patella tendon autograft. All surgery had been undertaken by experienced orthopaedic surgeons using standard procedures, with none of the cases having any secondary procedures, beyond the primary ACLR. At the time of surgery none of these athletes had any significant meniscus lesions or chondral damage reported (as assessed either from MRI or by the orthopaedic surgeon at the time of surgery). Ethical approval was provided by the University's ethical committee and written informed consent was attained from all participants.

\section{Procedures}

Tests were undertaken in the following order: isokinetic test, isometric test, quadriceps activation test; hop tests with a 10 minute rest between each group of tests, to minimize any effect of fatigue. 
116 Isokinetic eccentric and concentric strength of the quadriceps was assessed using a Biodex isokinetic

117 dynamometer (Biodex Medical Systems, Shirley, NY, USA). The test was conducted at an angular velocity of $11860^{\circ} . \mathrm{sec}^{-1}$ through a range of $0-90^{\circ}$ knee flexion. The participant performed five practice repetitions followed 119 three minutes later by five maximal repetitions of consecutive maximal effort eccentric, followed by concentric, 120 quadriceps actions, the best trial (highest peak torque) was recorded from the five repetitions for the eccentric and concentric efforts, respectively. All data were gravity corrected and normalised against body mass (absolute torque / body mass: $\mathrm{Nm} / \mathrm{kg}$ ).

\section{Isometric quadriceps strength test}

The isometric test of quadriceps strength was performed with the participants seated in an isokinetic dynamometer and positioned in $90^{\circ}$ hip flexion $90^{\circ}$ knee flexion $(13,25)$. The participant performed five practice repetitions followed by five maximal (3-5 s) repetitions, the highest peak torque was recorded from the five repetitions and normalised against body mass.

\section{Quadriceps central activation ratio}

The muscular inhibition of the quadriceps (central activation ratio (CAR)) was assessed, during a maximal isometric contraction (MVIC) of the quadriceps with the interpolated twitch technique (21). The participants were seated in an isokinetic dynamometer and positioned in $90^{\circ}$ hip flexion $90^{\circ}$ knee flexion. Two electrodes (proximal: $50 \times 130 \mathrm{~mm}$, distal: $7.5 \times 100 \mathrm{~mm}$ ) (Axelgaard, Fallbrook, Ca, USA) were placed on the quadriceps muscle at one-third and two-thirds from the distance between the anterior superior iliac spine and the upper border of the patella. Arthrogenic muscle inhibition measurements were taken using a Digitimer high voltage stimulator (DS7AH Digitimer Ltd, Hertfordshire, England). The participant undertook a maximal voluntary isometric contraction, 5 seconds. During the MVIC contraction two single pulses of $200 \mu$ s duration, 200 Volt and $120 \mathrm{~mA}$ were triggered three times (beginning, mid and end of the contraction; approximately at 1, 3 and 5 seconds) manually by the investigator when the MVIC force had plateaued on the monitor. Thus, electrical twitches were evoked at rest and added during a MVIC. Before CAR analysis, torque data was low-pass filtered at $150 \mathrm{~Hz}$ using a second-order Butterworth filter. CAR was quantified by calculating the difference between the stimulus-evoked torque during MVIC to the stimulus-evoked torque at rest and expressed in \%: activation deficit (AD) at 100\% MVIC. The smaller the deficit, the less the inhibition, whereby an inhibition of $0 \%$ means that the subject was able to fully recruit the muscle without showing any signs of inhibition. 
146 Hop tests: the assessment of single hop for distance (SHD) and cross over hop for distance (CHD) was 147 undertaken $(11,17)$. For the single hop, subjects were required to hop forwards as far as possible along the line 148 of the standard tape measure and land on the same limb. In the crossover hop subjects maximally hopped forward 4 times, alternately crossing two parallel lines $15 \mathrm{~cm}$ apart, therefore participating in two medial and two lateral direction landings. In both cases distance was measured from the start line to the rear of the foot upon final landing. The distance hopped was then normalized to a percentage of leg length, by dividing the distance hopped by the participant's leg length (distance anterior superior iliac spine to medial malleolus) and multiplying by 100 . The participants performed 3 practice trials and then a test trial as per the method of Munro and Herrington (18).

\section{Statistical Analyses}

Reliability of measures: Eight uninjured semi-professional footballers (age 20.1 \pm 5.3 years, body mass $85.0 \pm$ $10.1 \mathrm{~kg}$, height $1.73 \pm 0.2 \mathrm{~m}$, and a global KOOS questionnaire score of $94.8 \pm 4.3$ at time of testing), undertook all the tests on two separate occasions to assess the reliability of the tests. The reliability of the tests was assessed using intraclass correlation coefficient (ICC model 2,1), standard error of measurement (SEM) and coefficient of variance $(\% \mathrm{CV})$. Intraclass correlation coefficients were interpreted using established criteria (3) as follows: Poor - <0.40; Fair - 040-0.70; Good - 0.70-0.90; and excellent >0.90 and \%CV <10\% were regarded as acceptable (3).

Main analysis: Data were assessed for normality using Shapiro-Wilkes test. For each test ACLR and contralateral limb performance was then compared using individual paired t-tests with Cohen's $d$ effect sizes calculated and interpreted with 0.5 and below being a small effect size; greater than 0.5 being a medium effect size; 0.8 and above being a large effect size (3). Limb symmetry index was calculated by dividing the ACLR limb performance by the contralateral limb performance and multiplying by 100 to give a percentage. A prior power analysis $\left({ }^{*} \mathrm{G} *\right.$ Power, Version 3.1 .7$)$ with mean differences between two dependent means were used to calculate the required sample size, with an effect size of 2.53 (peak isometric force, ACLR and noninjured knee) and an alpha level of $p \leq 0.05,10$ subjects were required to obtain power of 1.0

\section{Results}

174 The global KOOS score for the ACLR group was $89.9 \pm 5.1$, whilst the score from the uninjured group used for the reliability study was $94.8 \pm 4$. 
Table 1: Reliability of dependent variable

\begin{tabular}{|c|c|c|c|}
\hline Test & ICC $(95 \% \mathrm{CI})$ & SEM* & $\% \mathrm{CV}$ \\
\hline Knee extensors eccentric peak torque $\left(\mathrm{Nm} . \mathrm{kg}^{-1}\right)$ & $\begin{array}{c}0.76 \\
(0.52-0.91)\end{array}$ & 0.48 & 14.3 \\
\hline Knee extensors concentric peak torque $\left(\mathrm{Nm} \mathrm{kg}^{-1}\right)$ & $\begin{array}{c}0.87 \\
(0.66-0.95)\end{array}$ & 0.45 & 13.0 \\
\hline Knee extensors Isometric peak force $\left(\mathrm{Nm} \cdot \mathrm{kg}^{-1}\right)$ & $\begin{array}{c}0.97 \\
(0.91-0.99)\end{array}$ & 0.23 & 6.9 \\
\hline Central activation ratio $(\%)$ & $\begin{array}{c}0.89 \\
(0.7-0.96) \\
\end{array}$ & 2.3 & 9.8 \\
\hline Single hop for distance (\% leg length) & $\begin{array}{c}0.85 \\
(0.61-0.95)\end{array}$ & 7.9 & 7.8 \\
\hline Cross over hop for distance (\% leg length) & $\begin{array}{c}0.8 \\
(0 .-0.93)\end{array}$ & 19.7 & 8.5 \\
\hline
\end{tabular}

ICC = intraclass correlation coefficient; $\mathrm{CI}=$ confidence interval; SEM = standard error of measurement; *SEM is presented for absolute units; $\mathrm{CV}=$ coefficient of variation

All tests show good reliability, however eccentric and concentric knee extensor strength exceeded the acceptable levels of variability (Table 1$)$.

Medium to large significant differences in quadriceps strength (eccentric, concentric and isometric) were found between and the difference was greater than the SEM, with the uninjured limb performing consistently better than the ACLR limb. There was a large and significant difference in the CAR between limbs which was greater than the SEM. Single hop distance showed a medium and significant difference between limbs, but only had a $64 \%$ chance of being superior based on the medium effect size, with a the difference being greater than the SEM. There was a small yet significantly greater CHD in the contralateral limb, with a difference greater than the SEM.

Table 2: Comparison between ACLR and non-injured legs across tests

\begin{tabular}{|c|c|c|c|c|c|}
\hline Test & Limb & Mean (SD) & Range & $p$ & $d$ \\
\hline \multirow{2}{*}{$\begin{array}{l}\text { Knee extensors } \\
\text { eccentric peak } \\
\text { torque }\left(\mathrm{Nm} . \mathrm{kg}^{-1}\right)\end{array}$} & ACLR & $3.28(0.79)$ & 1.89-4.72 & \multirow{2}{*}{0.0001} & \multirow{2}{*}{0.84} \\
\hline & Non-injured & $3.97(0.83)$ & $2.48-5.5$ & & \\
\hline \multirow{2}{*}{$\begin{array}{l}\text { Knee extensors } \\
\text { concentric peak } \\
\text { torque }\left(\mathrm{Nm} . \mathrm{kg}^{-1}\right)\end{array}$} & ACLR & $2.76(0.55)$ & $1.76-4.31$ & \multirow{2}{*}{0.0001} & \multirow{2}{*}{0.99} \\
\hline & Non-injured & $3.37(0.68)$ & $2.15-5.0$ & & \\
\hline \multirow{2}{*}{$\begin{array}{l}\text { Knee extensors } \\
\text { Isometric peak } \\
\text { force }\left(\mathrm{Nm} . \mathrm{kg}^{-1}\right)\end{array}$} & ACLR & $2.9(0.2)$ & 2.6-3.7 & \multirow{2}{*}{0.0001} & \multirow{2}{*}{2.53} \\
\hline & Non-injured & $3.7(0.4)$ & $3.0-4.8$ & & \\
\hline \multirow{2}{*}{$\begin{array}{l}\text { Central activation } \\
\text { ratio }(\%)\end{array}$} & ACLR & $18.8(7.9)$ & 11.1-32 & \multirow{2}{*}{0.0038} & \multirow{2}{*}{2.14} \\
\hline & Non-injured & $4.6(5.1)$ & 0-13 & & \\
\hline \multirow{2}{*}{$\begin{array}{l}\text { Single hop for } \\
\text { distance (\% leg } \\
\text { length) }\end{array}$} & ACLR & $183.9(26.1)$ & $141-226$ & \multirow{2}{*}{0.0001} & \multirow[b]{2}{*}{0.53} \\
\hline & Non-injured & $197.7(26.1)$ & $145-247$ & & \\
\hline
\end{tabular}




\begin{tabular}{lccccc}
$\begin{array}{l}\text { Cross over hop for } \\
\text { distance }(\% \text { leg }\end{array}$ & ACLR & $692.0(128.7)$ & $550-974$ & & \\
\cline { 2 - 4 } $\begin{array}{l}\text { length })\end{array}$ & Non-injured & $741.1(129.6)$ & $560-1012$ & & 0.38 \\
\hline
\end{tabular}

189

The limb symmetry index (LSI) percentages for all tasks along with the percentage of players, who achieved a LSI greater than the typical $90 \%$ cut off, are presented in table 3, highlighting that with the exception of the two hops tests low percentages of the players achieved the recommended level of LSI i.e. greater than $90 \%$.

\begin{tabular}{lccc}
\hline Test & $\begin{array}{c}\text { Mean }(\mathrm{SD}) \\
\%\end{array}$ & $\begin{array}{c}\text { Range } \\
\%\end{array}$ & $\begin{array}{c}\text { Percentage players } \\
\text { LSI }>90 \%(\mathrm{n}=)\end{array}$ \\
\hline Knee extensors eccentric peak torque & $\mathbf{8 2 . 8 ( 1 0 )}$ & $\mathbf{5 9 . 7 - 9 8 . 3}$ & $20(3)$ \\
\hline Knee extensors concentric peak torque & $\mathbf{8 3}(13.7)$ & $52.1-101.1$ & $20(3)$ \\
\hline Knee extensors Isometric peak force & $\mathbf{8 0 . 7}(7.8)$ & $63.7-96.8$ & $13(2)$ \\
\hline Single hop for distance & $\mathbf{9 3 . 2}(7.4)$ & $71.4-105.3$ & $67(10)$ \\
\hline Cross over hop for distance & $\mathbf{9 3 . 6}(7.6)$ & $\mathbf{6 6 . 9 - 1 1 3 . 8}$ & $73(11)$ \\
\hline Central activation ratio & $\mathbf{8 5 . 1}(5.6)$ & $75.5-92.1$ & $13(2)$ \\
\hline
\end{tabular}

\section{Discussion}

This study has presented quadriceps strength and hop performance data from a group of professional footballers following ACLR who had all been cleared to return to play. This is the first time data of this type has been present for such high level professional footballers. Because of the unique nature of this group, data from uninjured players is limited or not available. This study's data has been presented with effect sizes for the differences and the SEM for all tests, in order to give the findings some context. Despite being cleared to play, the majority of these individuals showed moderate to large significant deficits in both their quadriceps strength and hop performance when compared to the uninjured leg. These differences are also reflected in the high percentage of individuals who failed to achieve a 90\% LSI score for the tests. Gokeler et al. (9) using similar test battery on a more general population of ACLR patients, found that for hop tests $78.5 \%$ patients passed LSI $>90 \%$ for SHD, but only $39.3 \%$ passed LSI $>90 \%$ for quadriceps concentric contraction, whilst only $35.7 \%$ patients had $>3.0 \mathrm{Nm} \cdot \mathrm{kg}^{-1}$ isometric quadriceps strength for the involved ACLR limb. Wellsandt et al. (22) reported that $23 \%$ of ACLR patients assessed at 6 months post operation failed to have isometric quadriceps strength LSI greater than $90 \%$, with $26 \%$ on SHD and $17 \%$ on CHD also failing to achieve an LSI greater than $90 \%$. Both of these studies reflect the results found in this study despite differing populations. Overall it would appear that despite being deemed fit to return to play significant deficits exist in strength and to a lesser extent hop performance in a variety of populations at the time of return to sport. 
213 The use of the contralateral (non-injured) leg as a performance comparison has been questioned, because it may

214 underestimate the true level of deficit. The contralateral leg of the ACLR patient has frequently been found to be 215 significantly weaker when compared to a control limb in un-injured individuals (4). This is highlighted in the 216 Wellsandt et al. (22) study, when the ACLR limb was compared to the contralateral leg performance scores 217 measured pre-operatively $37 \%$ failed to have a LSI greater than $90 \%$ for quadriceps strength, and when SHD \& 218 CHD were assessed $26 \%$ failed to achieve an LSI greater than $90 \%$. The levels of strength and hop performance of the contralateral limb in this study would appear not to fit this pattern, with performance in line or superior too previously reported values for non-injured limbs (23). Furthermore, in a group of similar strength levels, Zult et al. (26) found the uninjured contralateral leg to have had similar levels of strength to those of controls. Currently, little data is available to determine what might be an acceptable absolute level of strength, both Gokeler et al (9) and Pietrosimone et al (19) proposed $>3.0 \mathrm{Nm} \cdot \mathrm{kg}^{-1}$ isometric quadriceps strength. Comparable data for eccentric and concentric strength would appear not to be available with little normative data present in the literature (in elite sportsman) to guide the decision making process.

This study does call into question only using functional tests such as hop tests and questionnaires such as KOOS in isolation without also measuring strength. The majority of participants (greater than 2/3) had LSI for hop tests of greater than $90 \%$ and global KOOS score over 90 which have been regarded a sufficient level of functional performance for return to sport. The findings of this study then support those of Gokeler et al. (9) that a battery of tests is required including quadriceps strength to define a patient's readiness to return to sport. The results of this study also call into question the continued reporting of LSI without also presenting absolute strength scores, both would appear to be required to give a full picture of performance (9).

The absolute level of quadriceps strength and limb asymmetry, have both been associated with the level of long term functional performance $(8,19,23)$. Furthermore, deficits in quadriceps strength have been related to the development of OA in an ACLR population (6). As ongoing knee symptoms and OA occur frequently in this population (5), it might be that the ongoing deficits in quadriceps performance might be related to this occurrence (1). A second ACL injury (either contralateral or graft rupture) is another relatively high frequency occurrence in this population, especially in the under 20 year old's (21). Pietrosimone et al (19 reported isometric quadriceps strength $>3.1 \mathrm{Nm} \cdot \mathrm{kg}^{-1}$ increased the chances of achieving acceptable levels of self-reported outcome by 8.15 times (specificity 0.84 , sensitivity 0.61 ). Whilst having an LSI $\geq 96.5 \%$ for isometric quadriceps strength increased the likelihood of reporting an acceptable outcome by 2.78 times (specificity 0.70 , 
sensitivity 0.55 ). Wellsandt et al. (21) reported that failure to achieve a LSI of greater than $90 \%$ for quadriceps strength, gave a specificity of 0.31 and sensitivity of 0.82 for predicting a second ACL injury. Poor quadriceps performance at return to play could therefore be related to increased risk of further ACL injury, ongoing knee symptoms and degenerative joint disease. What is unclear from the retrospective studies into ACLR outcome in football is if players had ongoing issues, as none of these studies $(7,20,25)$ reported levels of symptoms through tools such as functional questionnaires. They do report around a 1/3 of players are not playing at the same level, which coincides with the figure reported by Mai et al. (17) for American professional sports, but all of these studies fail to indicate the reasons for this.

The levels of quadriceps inhibition in the ACLR leg reported in this paper are similar to those previously reported by Kuenze et al (13). The level of quadriceps inhibition has been shown to be significantly related to the level of quadriceps strength (15), so may in part provide an explanation for the differences between the ACLR and the non ACLR limbs.

A strength of this paper is that it that it presents data on a unique population, this is obviously also a limitation as the findings might not be applicable to other sports or non-elite athletes. But because of the full time professional nature of these athletes it could be expected that other athletes with less support through rehabilitation, may not do as well as these individuals and have poorer results. Another limitation is that no detail was presented on the specific elements of the rehabilitation these athletes undertook, future study should identify if the inclusion (or exclusion) of specific exercises and activities has a significant impact on results. The study also only included individuals who had had isolated ACL injury, this obviously limits applicability. As more extensive damage to the knee is likely to create greater levels of inhibition, it is unlikely that these findings will be reversed in other populations and may even be accentuated. Finally, this study presents no follow up on these athletes so the impact of these findings on future sporting performance and development of comorbidities is unknown, future studies should attempt to track these individuals to understand the impact of findings. Furthermore, it is also not known if these deficits will change over time, some parameters such as rate of force development appear to normalize after 12 months (3), whilst others such as strength do not (15). Though in reality in the world of professional sport once findings such as these are identified it is unlikely that there will be an attempt to address them, so confounding any follow up. 
274 The retrospective studies into the professional sports, would appear to indicate that a high proportion of players return to play at the same level following ACLR, but a significant number are not performing at that level within 3 years of surgical repair of their ACL. The findings of this paper demonstrate significant deficits in quadriceps strength and activation and to a lesser extent performance during hop tests, despite the players being deemed fit to return to play. It might be hypothesised that there could be a link between the findings of this paper and the players who fail to maintain their level of performance or develop secondary issues. If this proves to be the case, then significant attention should be paid to re-establishing full quadriceps activation and strength prior to returning to unrestricted sporting activity.

Compliance with Ethical Standards: All procedures performed in studies involving human participants were in accordance with the ethical standards of the institutional research committee and with the 1964 Helsinki declaration and its later amendments or comparable ethical standards. This study was approved by University Research Ethics and Governance Committee (ref: HSCR14/68). All participants gave written informed consent.

Conflict of interest: All authors declare that they have no conflicts of interest.

Funding: No funding was received for this study.

1. Amin S, Baker K, Niu J. (2009) Quadriceps strength and the risk of cartilage loss and symptom progression in knee osteoarthritis. Arthritis Rheum. 60:189-198.

2. Adern C, Taylor N, Feller J, Webster K. (2014) Fifty five percent return to sport following anterior cruciate ligament reconstruction surgery: an updated systematic review and meta-analysis including development as an adjunctive outcome measure for return-to-sport decisions after anterior cruciate ligament reconstruction. J Orthop Sports Phys Ther 42:772-80. 
4. Batterham A, George K. (2003) Reliability in evidence-based clinical practice: a primer for allied health professionals Phys Ther Sport 4:122-128

5. Chung K, Ha J, Teom C, Ra H, Lim J, Kwon J, Kim J. (2015) Are muscle strength and function of the lower limb weakened after anterior cruciate ligament injury?: A two year follow up after reconstruction. Am J Sports Med 43:3013-3021

6. Culvenor A, Cook J, Collins N, Crossley K. (2013) Is patellofemoral joint osteoarthritis an underrecognised outcome of anterior cruciate ligament reconstruction? A narrative literature review. Brit J Sports Med 47:66-70.

7. Culvenor A, Ruhdorfer A, Carsten J, Eckstein F, Oiestad B. (2017) Knee extensor strength and risk of structural, symptomatic and functional decline in knee osteoarthritis: a systematic review and metaanalysis. Arthritis Care Res 69:649-658

8. Erickson B, Harris J, Cvetanovich G. (2013) Performance and return to sport after anterior cruciate ligament reconstruction in male Major League Soccer players. Orthop J Sports Med 1:23-25.

9. Flosadottir V, Roos E, Ageberg E. (2016) Muscle function is associated with future patient reported outcomes in young adults with ACL injury. BMJ Open Sport Exs Med 10 DOI:10.1136/bmjsem-2016000154

10. Gokeler A, Welling W, Zaffagnini S, Seil R, Padua D. (2016) Development of a test battery to enhance safe return to sports after anterior cruciate ligament reconstruction. Kn surg sports traumatol arthros $25 ; 192-195$

11. Herrington L, Comfort P. (2013) Training for prevention ACL injury: incorporation landing skill challenges into a program Stren Cond J 35:59-65

12. Herrington L, Myer G, Horsley I. (2013) Task based rehabilitation protocol for elite athletes following Anterior Cruciate ligament reconstruction: a clinical commentary Phys Ther Sport 14;188-198

13. Kuenze C, Hertel J, Weltman A, Diduch D, Saliba S, Hart J. 2015 Persistent neuromuscular and corticomotor quadriceps asymmetry after anterior cruciate ligament reconstruction. J Athl Train 50;303312

14. Lai C, Ardern C, Feller J, Webster K. (2017) Eighty three percent of elite athletes return to preinjury sport after anterior cruciate ligament reconstruction: a systematic review with meta-analysis of return to sport rates, graft rupture rates and performance outcomes. Brit J Sports Med DOI: 10.1136/bjsports-2016096836 
15. Lepley A, Ericksen H, Sohn D, Pietrosimone B. 2014 Contributions of Neural Excitability and Voluntary Activation to Quadriceps Muscle Strength Following Anterior Cruciate Ligament Reconstruction, Knee 21;736-742

16. Logerstedt D, Di Stasi D, Grundem H, Lynch A, Eitzen I, Engebretsen L, Risberg M, Axe M, SnyderMackler L. (2014) Self reported knee function can identify athletes who fail return to activity criteria up to 1 year after anterior cruciate ligament reconstruction. A Delaware-Oslo ACL cohort study. J Orthop Sports Phys Ther 44:914-923

17. Mai H, Chun D, Schneider A, Erickson B, Freshman R, Kester B, Verma N, Hsu W. (2017) Performance based outcomes after anterior cruciate ligament reconstruction in professional athletes differ between sports Am J Sports Med 45;2226-2231

18. Munro A, Herrington L. (2011) Between session reliability of four hop tests and the agility T-test J Stren Cond Res 25;1470-1477

19. Pietrosimone B, Lepley A, Harkey M, Luc B, Blackburn T, Gribble P, Spang J, Sohn D. (2017) Quadriceps strength predicts self-reported function post ACL reconstruction Med Sci Sport Exs 48;16711677

20. Risberg A, Grinde H, Oiestad B. (2016) We need to implement current evidence in the early rehabilitation programs to improve long term outcome after ACLR. J Orthop Sports Phys Ther 9,710-713

21. Walden M, Hagglund M, Magnusson H, Ekstrand J. (2016) ACL injuries in men's professional football: a 15 year prospective study on time trends and return to play rates reveals only $65 \%$ of players still play at top level 3 years after ACL rupture. Brit J Sports Med 50;744-750

22. Webster K, Feller J, Leigh W. (2014) Younger patients are at increased risk for graft rupture and contralateral injury after anterior cruciate ligament reconstruction. Am J Sports Med 42:641-7

23. Wellsandt E, Failla M, Synder-Mackler L. (2017) Limb symmetry indexes can overestimate knee function after anterior cruciate ligament injury. J Orthop Sports Phys Ther 47:334-338

24. Willigenburg N, McNally M, Hewett T. (2014) Quadriceps and Hamstring strength in athletes. Chp 2 in Kaeding, C., Borchers, J. (Eds) Hamstring and Quadriceps injuries in athletes. A clinical guide. Springer, New York. ISBN: 978-1-4899-7509-6

25. Zaffagnini S, Grassi A, Marcheggiani Muccioli G. (2014) Return to sport after anterior cruciate ligament reconstruction in professional soccer players. Knee 21:731-5. 
361 26. Zult T, Gokeler A, van Raay J, Brouwer R, Zijdewind I, Hortobagyi T. (2016) An anterior cruciate ligament injury does not affect the neuromuscular function of the non-injured leg except for dynamic balance and voluntary quadriceps activation. Kn surg sports traumatol arthros 25;172-183 